\title{
Form factors of twist fields in the lattice Dirac theory
}

\author{
P Gavrylenko ${ }^{1}$, N Iorgov $^{2}$ and O Lisovyy ${ }^{3}$ \\ ${ }^{1}$ Department of Physics, Kyiv National University, 03022 Kyiv, Ukraine \\ 2 Bogolyubov Institute for Theoretical Physics, 03680 Kyiv, Ukraine \\ ${ }^{3}$ Laboratoire de Mathématiques et Physique Théorique CNRS/UMR 6083, \\ Université de Tours, Parc de Grandmont, 37200 Tours, France \\ E-mail: iorgov@bitp.kiev.ua, lisovyi@lmpt.univ-tours.fr
}

\begin{abstract}
We study $U(1)$ twist fields in a two-dimensional lattice theory of massive Dirac fermions. Factorized formulas for finite-lattice form factors of these fields are derived using elliptic parametrization of the spectral curve of the model, elliptic determinant identities and theta functional interpolation. We also investigate the thermodynamic and the infinite-volume scaling limit, where the corresponding expressions reduce to form factors of the exponential fields of the sine-Gordon model at the free-fermion point.

PACS numbers: $05.50+\mathrm{q}, 02.30 \mathrm{Ik}$
\end{abstract}

\section{Introduction}

It is a general property of two-dimensional quantum field theories that their symmetries give rise to new local fields, whose correlation functions are nontrivial even if the underlying theory is free. Two paradigmatic examples are given by the disorder variables in the Ising field theory [16, 30] and $U(1)$ twist fields in the massive Dirac theory [23, 29, 31, directly related to the exponential fields in the sine-Gordon model at the free-fermion point.

Correlation functions of twist fields in the Dirac theory, as well as in its generalizations to curved space and non-zero background magnetic field [6, 17, 18, 27], are also interesting from the mathematical point of view. They satisfy nonlinear differential equations [1, 8, 29], which in the simplest cases can be solved in terms of Painlevé functions. The knowledge of the long- and short-distance behaviour of the twopoint correlators provides solutions to nontrivial asymptotic and connection problems of Painlevé theory. Recently, it has also been observed [19] that such correlators coincide with the gap probabilities for the classical kernels arising in the representation theory of big groups [2].

The aim of this paper is to construct lattice analogs of $U(1)$ twist fields in the Dirac model, satisfying the following properties: (i) they should be defined via the branching 
of lattice fermion fields, (ii) one should be able to calculate their form factors explicitly and (iii) these form factors should reproduce the known expressions in the scaling limit. Besides full control of the theory, such an integrable finite-lattice regularization can be used for investigations at non-zero temperature and for a mathematically sound derivation of the relative normalization of conformal and infrared asymptotics of the two-point correlator [5, 21]. It may also be instrumental in going beyond the freefermion point.

While the Ising field theory possesses a natural lattice regularization, only a few results are available in the Dirac case. First attempt to introduce twist fields on the infinite lattice was made in [25]. The corresponding definition was supported by the computation of the vacuum expectation value (reproducing the expected scaling dimension), and was further strengthened by the analysis of correlations at the critical point [26]. Another, seemingly unrelated definition was used in [5, 20] to derive a number of determinant representations for the two-point function of lattice twist fields. The present work is devoted to the computation of their form factors, i. e. matrix elements of the field operators in the basis of transfer matrix eigenstates.

The paper is planned as follows. In Subsections 2.1 and 2.2, we introduce a oneparameter generalization of the lattice Dirac operator considered in [5, 20] and explain the definition of twist fields in the functional integral framework. Transition to the operator formalism is performed in the next subsection. Using the coherent states approach, the transfer matrix and the twist field operator are written as exponentials of fermion bilinears, see formulas (9) and (11) below. Subsection 2.4 is devoted to the construction of multiparticle Fock states simultaneously diagonalizing the transfer matrix and the operator of translations.

In Subsection 3.1, it is explained how form factors of twist fields can be found from the linear transformations relating fermionic creation-annihilation operators of different periodicity. In particular, the vacuum expectation value and two-particle form factors are expressed in terms of two square matrices $C$ and $D$ of dimension equal to the lattice size. Essentially, one needs to compute the quantities $D^{-1}, D^{-1} C$ and det $D$, cf. (25)-(32). This task is solved in Subsection 3.2 by first noting that in the elliptic parametrization of the spectral curve of the model $C$ and $D$ are given (up to diagonal factors) by elliptic Cauchy matrices, and then using Frobenius determinant identity and theta functional interpolation along the lines of [13]. The corresponding expressions are further simplified in Subsection 3.3. Finite-lattice two-particle form factors are given by (42), (44) and (45), and the multiparticle ones have the factorized form (46), (49)). These formulas represent the main result of the paper. In Subsection 3.4 we analyze the thermodynamic (infinite-lattice) limit. The final answer has a remarkably simple expression in terms of the Jacobi theta functions, see (54)-(56). We remark that the vacuum expectation value (54) reproduces the earlier result of [25]. Field theory limit is considered in Subsection 3.5. It is shown that the scaled form factors coincide with those of the exponential fields of sine-Gordon model at the free-fermion point [1, 23, 31]. We conclude with a brief discussion of results and open problems. 
Form factors of twist fields in the lattice Dirac theory

\section{Lattice Dirac theory}

\subsection{Fermions}

Let $\psi, \bar{\psi}$ denote two 2-component Grassmann fields on an $M \times N$ square lattice. Consider the standard free-fermion action $S[\psi, \bar{\psi}]=\bar{\psi} D \psi$, where the lattice Dirac operator is chosen as

$$
D=\frac{1}{c_{x}^{*}}\left(\begin{array}{cc}
c_{x}^{*} s_{y}-s_{x}^{*} c_{y} \nabla_{y} & -c_{y}+c_{x}^{*} \nabla_{x} \\
c_{y}-c_{x}^{*} \nabla_{-x} & c_{x}^{*} s_{y}-s_{x}^{*} c_{y} \nabla_{-y}
\end{array}\right)
$$

Here $\nabla_{x, y}$ denote the shifts by one lattice site in the horizontal and vertical directions, so that e. g. $\nabla_{x} \psi_{x, y}=\psi_{x+1, y}, \nabla_{y} \psi_{x, y}=\psi_{x, y+1}$. The boundary conditions with respect to $x$ and $y$ are antiperiodic and $\alpha$-periodic, respectively. This means that

$$
\left\{\begin{array} { l } 
{ \psi _ { x + M , y } = - \psi _ { x , y } , } \\
{ \overline { \psi } _ { x + M , y } = - \overline { \psi } _ { x , y } , }
\end{array} \quad \left\{\begin{array}{l}
\psi_{x, y+N}=e^{2 \pi i \alpha} \psi_{x, y} \\
\bar{\psi}_{x, y+N}=e^{-2 \pi i \alpha} \bar{\psi}_{x, y}
\end{array}\right.\right.
$$

The parameters $c_{i}, s_{i}, c_{i}^{*}, s_{i}^{*}(i=x, y)$ are expressed in terms of two constants $\mathcal{K}_{x, y} \in \mathbb{R}_{>0}$ as

$$
c_{i}=\cosh 2 \mathcal{K}_{i}, \quad s_{i}=\sinh 2 \mathcal{K}_{i}, \quad c_{i}^{*}=\cosh 2 \mathcal{K}_{i}^{*}, \quad s_{i}^{*}=\sinh 2 \mathcal{K}_{i}^{*},
$$

where the dual couplings are given by $\mathcal{K}_{i}^{*}=\operatorname{arctanh} e^{-2 \mathcal{K}_{i}}$. They satisfy Ising-type relations $s_{i}^{*}=s_{i}^{-1}, c_{i}^{*}=c_{i} s_{i}^{-1}$. It will be assumed in the following that $\mathcal{K}_{x}^{*}<\mathcal{K}_{y}$. Dirac operator considered in [5, 20] is obtained from (11) by setting $\mathcal{K}_{x}=\mathcal{K}_{y}$.

Fermion propagator can be found using Fourier transform. One obtains

$$
\left\langle\bar{\psi}_{x, y} \psi_{x^{\prime}, y^{\prime}}\right\rangle=\frac{1}{M N} \sum_{\phi, \theta} \frac{e^{i \phi\left(x-x^{\prime}\right)+i \theta\left(y-y^{\prime}\right)}}{2 c_{y}\left(\cosh \gamma_{\theta}-\cos \phi\right)}\left(\begin{array}{cc}
c_{x}^{*} s_{y}-s_{x}^{*} c_{y} e^{-i \theta} & c_{y}-c_{x}^{*} e^{i \phi} \\
-c_{y}+c_{x}^{*} e^{-i \phi} & c_{x}^{*} s_{y}-s_{x}^{*} c_{y} e^{i \theta}
\end{array}\right)
$$

where

$$
\cosh \gamma_{\theta}=c_{x}^{*} c_{y}-s_{x}^{*} s_{y} \cos \theta
$$

The multipoint correlations are readily computable from the Wick theorem. The summation in (2) is performed over $\phi=\frac{2 \pi}{M}\left(j+\frac{1}{2}\right), j=0, \ldots, M-1$ and $\theta=\frac{2 \pi}{N}(k+\alpha)$, $k=0, \ldots, N-1$. In the thermodynamic limit $M, N \rightarrow \infty$ one has $\frac{1}{M N} \sum_{\phi, \theta} \rightarrow$ $\frac{1}{(2 \pi)^{2}} \iint_{0}^{2 \pi} d \phi d \theta$ and the answer can be expressed in terms of elliptic integrals.

\subsection{Twist fields}

It is instructive to start with an example. Choose a closed path $\mathcal{P}$ on the dual lattice (Fig. 1) and make the transformation $\psi \mapsto e^{2 \pi i \nu} \psi, \bar{\psi} \mapsto e^{-2 \pi i \nu} \bar{\psi}$ with $\nu \in \mathbb{R}$ at all lattice sites inside this contour. Because of the global $U(1)$-symmetry, the action $S[\psi, \bar{\psi}]$ will change only at the edges intersected by $\mathcal{P}$.

If the corresponding changes are made along an open path $\mathcal{P}_{A B}$ joining two points $A$ and $B$ on the dual lattice, the resulting functional integral will depend on the positions 


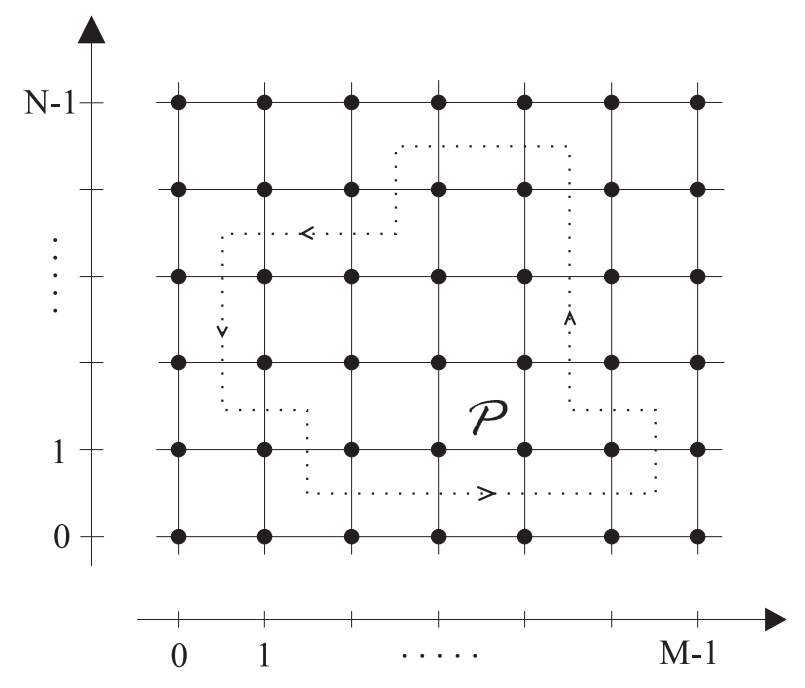

Fig. 1

of these points and homotopy class of the path, but not on its shape. One way to choose $\mathcal{P}_{A B}$ is shown in Fig. 2. In this case, the action is modified by

$$
\delta S_{A B}=\delta S_{A}+\delta S_{B}+\delta S_{\text {b.c. }}
$$

where $\delta S_{A, B}$ correspond to the vertical segments and $\delta S_{\text {b.c. }}$ to the horizontal one. Explicitly,

$$
\begin{gathered}
\delta S_{A}=2 i \sin \pi \nu \sum_{y^{\prime \prime}=0}^{y-1}\left(e^{i \pi \nu} \bar{\psi}_{x-1, y^{\prime \prime}}^{1} \psi_{x, y^{\prime \prime}}^{2}+e^{-i \pi \nu} \bar{\psi}_{x, y^{\prime \prime}}^{2} \psi_{x-1, y^{\prime \prime}}^{1}\right), \\
\delta S_{B}=-2 i \sin \pi \nu \sum_{y^{\prime \prime}=0}^{y^{\prime}-1}\left(e^{-i \pi \nu} \bar{\psi}_{x^{\prime}-1, y^{\prime \prime}}^{1} \psi_{x^{\prime}, y^{\prime \prime}}^{2}+e^{i \pi \nu} \bar{\psi}_{x^{\prime}, y^{\prime \prime}}^{2} \psi_{x^{\prime}-1, y^{\prime \prime}}^{1}\right), \\
\delta S_{\text {b.c. }}=-2 i \sin \pi \nu \frac{c_{y}}{c_{x}} \sum_{x^{\prime \prime}=x}^{x^{\prime}-1}\left(e^{i \pi\left(\alpha+\alpha^{\prime}\right)} \bar{\psi}_{x^{\prime \prime}, N-1}^{1} \psi_{x^{\prime \prime}, 0}^{1}-e^{-i \pi\left(\alpha+\alpha^{\prime}\right)} \bar{\psi}_{x^{\prime \prime}, 0}^{2} \psi_{x^{\prime \prime}, N-1}^{2}\right)
\end{gathered}
$$

with $\alpha^{\prime}=\alpha+\nu$. Without any loss of generality, we assume that $0 \leq \alpha, \alpha^{\prime}<1$.

Twist fields live on the dual lattice. Their two-point correlation function is defined as the normalized partition function of the Dirac theory with the defect contribution (4):

$$
\left\langle\mathcal{O}_{\alpha, \alpha^{\prime}}(A) \mathcal{O}_{\alpha^{\prime}, \alpha}(B)\right\rangle=\frac{\int \mathcal{D} \psi \mathcal{D} \bar{\psi} e^{S[\psi, \bar{\psi}]+\delta S_{A B}}}{\int \mathcal{D} \psi \mathcal{D} \bar{\psi} e^{S[\psi, \bar{\psi}]}}
$$

Note that the effect of $\delta S_{\text {b.c. }}$ amounts to the change of the vertical boundary conditions for fermions from the horizontal interval $\left[x, x^{\prime}-1\right]$ from $\alpha$ - to $\alpha^{\prime}$-periodic ones. More generally, the appearance of twist field $\mathcal{O}_{\alpha, \alpha^{\prime}}(A)$ in an arbitrary correlation function means that

- the vertical boundary conditions for fermions to the left and right of $A$ are $\alpha$ - and $\alpha^{\prime}$-periodic, respectively; 


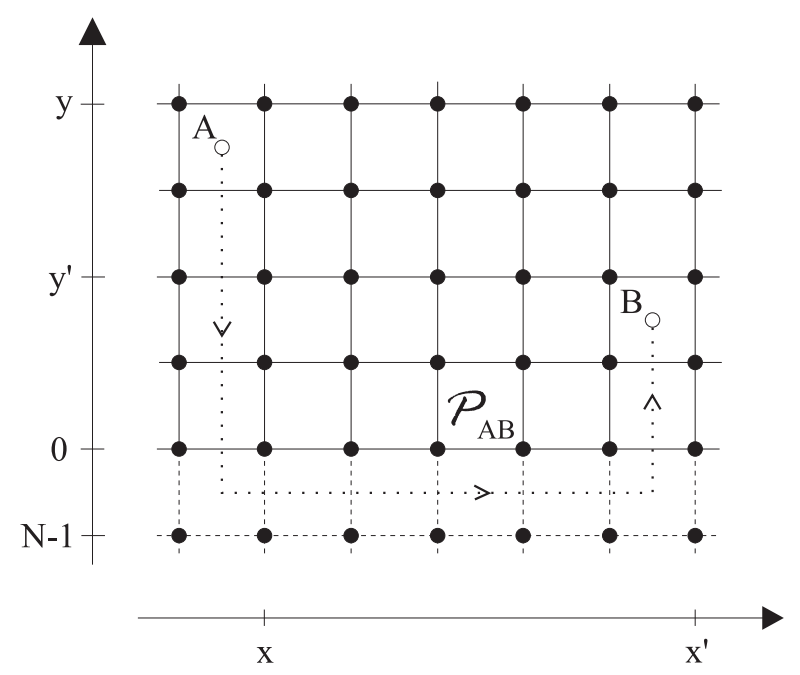

Fig. 2

- the term $\delta S_{A}$ should be added to the action.

In the heuristic continuum limit, this corresponds to integrating over field configurations having counterclockwise monodromy $e^{2 \pi i \nu}$ of $\psi$ (resp. $e^{-2 \pi i \nu}$ for $\bar{\psi}$ ) around $A$.

\subsection{Coherent states and the operator formalism}

Locality of twist fields becomes manifest in the operator formalism, which also provides a convenient framework for the computation of correlation functions.

Let us introduce two sets of fermionic creation-annihilation operators satisfying canonical anticommutation relations

$$
\left\{a_{y}, a_{y^{\prime}}^{\dagger}\right\}=\left\{b_{y}, b_{y^{\prime}}^{\dagger}\right\}=\delta_{y y^{\prime}}, \quad y, y^{\prime}=0, \ldots, N-1,
$$

with all other anticommutators vanishing. Define in the usual way the vacuum vectors $\langle v a c|$ and $|v a c\rangle$, normalized as $\langle v a c \mid v a c\rangle=1$, and the corresponding $2^{2 N}$-dimensional Fock space $\mathcal{F}$. Further, introduce the coherent states

$$
\begin{aligned}
\left|\boldsymbol{\psi}_{x}^{1}, \overline{\boldsymbol{\psi}}_{x}^{1}\right\rangle & =\exp \sum_{y=0}^{N-1}\left(a_{y}^{\dagger} \bar{\psi}_{x, y}^{1}+b_{y}^{\dagger} \psi_{x, y}^{1}\right)|v a c\rangle, \\
\left\langle\boldsymbol{\psi}_{x}^{2}, \overline{\boldsymbol{\psi}}_{x}^{2}\right| & =\langle v a c| \exp \sum_{y=0}^{N-1}\left(\psi_{x, y}^{2} a_{y}+\bar{\psi}_{x, y}^{2} b_{y}\right),
\end{aligned}
$$

where $\left\{\psi_{x, y}^{i}\right\},\left\{\bar{\psi}_{x, y}^{i}\right\}$ denote Grassmann variables anticommuting with all creationannihilation operators. These states satisfy the following standard properties:

- For $y=0, \ldots, N-1$ one has

$$
\left\{\begin{array}{l}
a_{y}\left|\boldsymbol{\psi}_{x}^{1}, \overline{\boldsymbol{\psi}}_{x}^{1}\right\rangle=\bar{\psi}_{x, y}^{1}\left|\boldsymbol{\psi}_{x}^{1}, \overline{\boldsymbol{\psi}}_{x}^{1}\right\rangle \\
b_{y}\left|\boldsymbol{\psi}_{x}^{1}, \overline{\boldsymbol{\psi}}_{x}^{1}\right\rangle=\psi_{x, y}^{1}\left|\boldsymbol{\psi}_{x}^{1}, \overline{\boldsymbol{\psi}}_{x}^{1}\right\rangle
\end{array}\right.
$$


Form factors of twist fields in the lattice Dirac theory

$$
\left\{\begin{array}{l}
\left\langle\psi_{x}^{2}, \overline{\boldsymbol{\psi}}_{x}^{2}\right| a_{y}^{\dagger}=\left\langle\boldsymbol{\psi}_{x}^{2}, \overline{\boldsymbol{\psi}}_{x}^{2}\right| \psi_{x, y}^{2} \\
\left\langle\boldsymbol{\psi}_{x}^{2}, \overline{\boldsymbol{\psi}}_{x}^{2}\right| b_{y}^{\dagger}=\left\langle\boldsymbol{\psi}_{x}^{2}, \overline{\boldsymbol{\psi}}_{x}^{2}\right| \bar{\psi}_{x, y}^{2}
\end{array}\right.
$$

- The scalar product of two coherent states is given by

$$
\left\langle\boldsymbol{\psi}_{x^{\prime}}^{2}, \overline{\boldsymbol{\psi}}_{x^{\prime}}^{2} \mid \boldsymbol{\psi}_{x}^{1}, \overline{\boldsymbol{\psi}}_{x}^{1}\right\rangle=\exp \sum_{y=0}^{N-1}\left(-\bar{\psi}_{x, y}^{1} \psi_{x^{\prime}, y}^{2}+\bar{\psi}_{x^{\prime}, y}^{2} \psi_{x, y}^{1}\right) .
$$

- The identity operator can be represented as a $4 N$-fold Grassmann integral

$$
\begin{aligned}
\mathbf{1}_{\mathcal{F}}= & \int \mathcal{D} \boldsymbol{\psi}_{x}^{1} \mathcal{D} \overline{\boldsymbol{\psi}}_{x}^{1} \mathcal{D} \boldsymbol{\psi}_{x^{\prime}}^{2} \mathcal{D} \overline{\boldsymbol{\psi}}_{x^{\prime}}^{2}\left|\boldsymbol{\psi}_{x}^{1}, \overline{\boldsymbol{\psi}}_{x}^{1}\right\rangle\left\langle\boldsymbol{\psi}_{x^{\prime}}^{2}, \overline{\boldsymbol{\psi}}_{x^{\prime}}^{2}\right| \times \\
& \times \exp \sum_{y=0}^{N-1}\left(\bar{\psi}_{x, y}^{1} \psi_{x^{\prime}, y}^{2}-\bar{\psi}_{x^{\prime}, y}^{2} \psi_{x, y}^{1}\right) .
\end{aligned}
$$

- The trace of any operator $\mathcal{O}$ can be written as an integral of its matrix element in the basis of coherent states with a Gaussian kernel:

$$
\begin{aligned}
\operatorname{Tr} \mathcal{O} & =\int \mathcal{D} \boldsymbol{\psi}_{x}^{1} \mathcal{D} \overline{\boldsymbol{\psi}}_{x}^{1} \mathcal{D} \boldsymbol{\psi}_{x^{\prime}}^{2} \mathcal{D} \overline{\boldsymbol{\psi}}_{x^{\prime}}^{2}\left\langle\boldsymbol{\psi}_{x^{\prime}}^{2}, \overline{\boldsymbol{\psi}}_{x^{\prime}}^{2}|\mathcal{O}| \boldsymbol{\psi}_{x}^{1}, \overline{\boldsymbol{\psi}}_{x}^{1}\right\rangle \times \\
& \times \exp \sum_{y=0}^{N-1}\left(-\bar{\psi}_{x, y}^{1} \psi_{x^{\prime}, y}^{2}+\bar{\psi}_{x^{\prime}, y}^{2} \psi_{x, y}^{1}\right) .
\end{aligned}
$$

- Matrix elements $\left\langle\boldsymbol{\psi}_{x^{\prime}}^{2}, \overline{\boldsymbol{\psi}}_{x^{\prime}}^{2}|\mathcal{O}| \boldsymbol{\psi}_{x}^{1}, \overline{\boldsymbol{\psi}}_{x}^{1}\right\rangle$ can be obtained by writing $\mathcal{O}$ in normally ordered form, making therein the replacements

$$
a_{y} \rightarrow \bar{\psi}_{x, y}^{1}, \quad b_{y} \rightarrow \psi_{x, y}^{1}, \quad a_{y}^{\dagger} \rightarrow \psi_{x^{\prime}, y}^{2}, \quad b_{y}^{\dagger} \rightarrow \bar{\psi}_{x^{\prime}, y}^{2},
$$

and multiplying the result by (6) .

Now consider the operator

$$
\begin{aligned}
V_{\alpha}= & : \exp \sum_{y=0}^{N-1}\left\{b_{y}^{\dagger}\left(s_{y}-c_{x}^{-1} c_{y} \nabla_{-y}\right) a_{y}^{\dagger}+\left(c_{x}^{*-1} c_{y}-1\right)\left(a_{y}^{\dagger} a_{y}+b_{y}^{\dagger} b_{y}\right)+\right. \\
& \left.+a_{y}\left(s_{y}-c_{x}^{-1} c_{y} \nabla_{y}\right) b_{y}\right\}:
\end{aligned}
$$

where it is understood that

$$
\left(\begin{array}{c}
a_{y+N}^{\dagger} \\
b_{y+N}
\end{array}\right)=e^{2 \pi i \alpha}\left(\begin{array}{c}
a_{y}^{\dagger} \\
b_{y}
\end{array}\right), \quad\left(\begin{array}{c}
a_{y+N} \\
b_{y+N}^{\dagger}
\end{array}\right)=e^{-2 \pi i \alpha}\left(\begin{array}{c}
a_{y} \\
b_{y}^{\dagger}
\end{array}\right) .
$$

Rewrite the quantity $Z=\operatorname{Tr} V_{\alpha}^{M}$ using at the first stage (8) with $x=N-1, x^{\prime}=0$ to calculate the trace, and inserting $M-1$ resolutions of unity (7) (with $x=k-1$ and $x^{\prime}=k$ between the $k$-th and $(k+1)$-th factor of $\left.V_{\alpha}\right)$. Then, computing matrix elements of $V_{\alpha}$ in the basis of coherent states, the reader may easily check that $Z$ coincides with the partition function of the Dirac theory described by (11).

In fact $V_{\alpha}$ is the transfer matrix characterizing discrete time evolution of twist fields. Vertical defects of the action associated to them divide the horizontal axis into intervals. 
Form factors of twist fields in the lattice Dirac theory

The evolution in different intervals is governed by the matrices $V_{\alpha}$ with appropriate values of $\alpha$. Twist fields are represented by the operators

$$
\mathcal{O}_{\alpha, \alpha^{\prime}}\left(y^{*}\right)=\exp 2 \pi i \nu \sum_{y^{\prime}=0}^{y-1}\left(-a_{y^{\prime}}^{\dagger} a_{y^{\prime}}+b_{y^{\prime}}^{\dagger} b_{y^{\prime}}\right),
$$

where $y^{*}=y-\frac{1}{2}$. This can be seen by noticing that

$$
\begin{gathered}
\exp \left\{\delta S_{A}+\sum_{y^{\prime}=0}^{N-1}\left(\bar{\psi}_{x-1, y^{\prime}}^{1} \psi_{x, y^{\prime}}^{2}-\bar{\psi}_{x, y^{\prime}}^{2} \psi_{x-1, y^{\prime}}^{1}\right)\right\}=\int \mathcal{D} \boldsymbol{\xi} \mathcal{D} \overline{\boldsymbol{\xi}}\left\langle\boldsymbol{\xi}^{2}, \overline{\boldsymbol{\xi}}^{2}\left|\mathcal{O}_{\alpha, \alpha^{\prime}}\left(y^{*}\right)\right| \boldsymbol{\xi}^{1}, \overline{\boldsymbol{\xi}}^{1}\right\rangle \times \\
\quad \times \exp \sum_{y^{\prime}=0}^{N-1}\left(\bar{\psi}_{x-1, y^{\prime}}^{1} \xi_{y^{\prime}}^{2}-\bar{\xi}_{y^{\prime}}^{2} \psi_{x-1, y^{\prime}}^{1}+\bar{\xi}_{y^{\prime}}^{1} \psi_{x, y^{\prime}}^{2}-\bar{\psi}_{x, y^{\prime}}^{2} \xi_{y^{\prime}}^{1}\right)
\end{gathered}
$$

and repeating the procedure used above for the computation of $Z$. For example, twopoint correlator (5) can be expressed as

$$
\left\langle\mathcal{O}_{\alpha, \alpha^{\prime}}(A) \mathcal{O}_{\alpha^{\prime}, \alpha}(B)\right\rangle=Z^{-1} \operatorname{Tr}\left(\mathcal{O}_{\alpha, \alpha^{\prime}}\left(y^{*}\right) V_{\alpha^{\prime}}^{x^{\prime}-x} \mathcal{O}_{\alpha^{\prime}, \alpha}\left(y^{\prime *}\right) V_{\alpha}^{M-\left(x^{\prime}-x\right)}\right) .
$$

The problem of effective calculation of correlation functions of twist fields therefore reduces to the computation of form factors of the operator (11) between the eigenstates of $V_{\alpha}$ and $V_{\alpha^{\prime}}$. Observe that e. g. $\mathcal{O}_{\alpha, \alpha^{\prime}}\left(0^{*}\right)$ is given by the identity operator on $\mathcal{F}$. However, its form factors are nontrivial since the corresponding bra and ket states diagonalize different transfer matrices.

\subsection{Transfer matrix diagonalization}

Define Fourier transforms of the creation-annihilation operators:

$$
\begin{aligned}
& \left(\begin{array}{l}
a_{\theta}^{\dagger} \\
b_{\theta}
\end{array}\right)=\frac{1}{\sqrt{N}} \sum_{y=0}^{N-1}\left(\begin{array}{c}
a_{y}^{\dagger} \\
b_{y}
\end{array}\right) e^{-i \theta y}, \\
& \left(\begin{array}{c}
a_{\theta} \\
b_{\theta}^{\dagger}
\end{array}\right)=\frac{1}{\sqrt{N}} \sum_{y=0}^{N-1}\left(\begin{array}{c}
a_{y} \\
b_{y}^{\dagger}
\end{array}\right) e^{i \theta y},
\end{aligned}
$$

where $\theta$ belongs to the set $\boldsymbol{\theta}_{\alpha}=\left\{\frac{2 \pi}{N}(k+\alpha) \mid k=0, \ldots, N-1\right\}$. The only nonvanishing anticommutators are given by $\left\{a_{\theta}, a_{\theta^{\prime}}^{\dagger}\right\}=\left\{b_{\theta}, b_{\theta^{\prime}}^{\dagger}\right\}=\delta_{\theta \theta^{\prime}}$. The transfer matrix $V_{\alpha}$ is block-diagonal in the Fourier basis,

$$
\begin{aligned}
V_{\alpha}= & : \exp \sum_{\theta \in \boldsymbol{\theta}_{\alpha}}\left\{\left(s_{y}-c_{x}^{-1} c_{y} e^{-i \theta}\right) b_{\theta}^{\dagger} a_{\theta}^{\dagger}+\left(c_{x}^{*-1} c_{y}-1\right)\left(a_{\theta}^{\dagger} a_{\theta}+b_{\theta}^{\dagger} b_{\theta}\right)+\right. \\
& \left.+\left(s_{y}-c_{x}^{-1} c_{y} e^{i \theta}\right) a_{\theta} b_{\theta}\right\}:
\end{aligned}
$$

The conjugation of fermions by $V_{\alpha}$ induces linear transformations

$$
\begin{aligned}
& V_{\alpha}\left(\begin{array}{c}
a_{\theta}^{\dagger} \\
b_{\theta}
\end{array}\right) V_{\alpha}^{-1}=\Lambda(\theta)\left(\begin{array}{c}
a_{\theta}^{\dagger} \\
b_{\theta}
\end{array}\right), \\
& V_{\alpha}\left(\begin{array}{c}
a_{\theta} \\
b_{\theta}^{\dagger}
\end{array}\right) V_{\alpha}^{-1}=\left(\Lambda^{-1}\right)^{T}(\theta)\left(\begin{array}{c}
a_{\theta} \\
b_{\theta}^{\dagger}
\end{array}\right),
\end{aligned}
$$


Form factors of twist fields in the lattice Dirac theory

where $\Lambda(\theta)$ is a Hermitian matrix with unit determinant, explicitly given by

$$
\Lambda(\theta)=\frac{1}{c_{y}}\left(\begin{array}{cc}
c_{x}^{*}\left(c_{y}^{2}+s_{y}^{2}\right)-2 s_{x}^{*} s_{y} c_{y} \cos \theta & -c_{x}^{*} s_{y}+s_{x}^{*} c_{y} e^{i \theta} \\
-c_{x}^{*} s_{y}+s_{x}^{*} c_{y} e^{-i \theta} & c_{x}^{*}
\end{array}\right) .
$$

It can be brought to the diagonal form, $\Lambda(\theta)=U(\theta)\left(\begin{array}{cc}e^{-\gamma_{\theta}} & 0 \\ 0 & e^{\gamma_{\theta}}\end{array}\right) U^{\dagger}(\theta)$, by a unitary transformation. Here $\gamma_{\theta}$ is defined as the positive solution of (3), and the columns of $U(\theta)=\left(\begin{array}{rr}f_{1}(\theta) & -\bar{f}_{2}(\theta) \\ f_{2}(\theta) & \bar{f}_{1}(\theta)\end{array}\right)$ are the eigenvectors of $\Lambda(\theta)$ normalized so that $\left|f_{1}(\theta)\right|^{2}+\left|f_{2}(\theta)\right|^{2}=1$. The freedom in the choice of the phase can be used to set

$$
\left(\begin{array}{l}
f_{1}(\theta) \\
f_{2}(\theta)
\end{array}\right)=\frac{1}{2 \sqrt{c_{y}^{*}}}\left(\begin{array}{cc}
e^{\mathcal{K}_{y}^{*}} & -e^{-\mathcal{K}_{y}^{*}} \\
e^{-\mathcal{K}_{y}^{*}} & e^{\mathcal{K}_{y}^{*}}
\end{array}\right)\left(\begin{array}{l}
\chi_{\theta} \\
\chi_{-\theta}
\end{array}\right)
$$

where

$$
\chi_{\theta}=\left[\chi_{-\theta}\right]^{-1}=\left[\frac{\left(1-\alpha e^{i \theta}\right)\left(1-\beta e^{-i \theta}\right)}{\left(1-\beta e^{i \theta}\right)\left(1-\alpha e^{-i \theta}\right)}\right]^{\frac{1}{4}}
$$

and $\alpha=\tanh \mathcal{K}_{x}^{*} \operatorname{coth} \mathcal{K}_{y}, \beta=\tanh \mathcal{K}_{x}^{*} \tanh \mathcal{K}_{y}$. Note that under the above conventions one has $\beta<\alpha<1$. Root function in (13) is taken on the principal branch.

A new set of the creation-annihilation operators

$$
\left(\begin{array}{c}
c_{\theta}^{\dagger} \\
d_{\theta}
\end{array}\right)=U^{\dagger}(\theta)\left(\begin{array}{c}
a_{\theta}^{\dagger} \\
b_{\theta}
\end{array}\right), \quad\left(\begin{array}{c}
c_{\theta} \\
d_{\theta}^{\dagger}
\end{array}\right)=U^{T}(\theta)\left(\begin{array}{c}
a_{\theta} \\
b_{\theta}^{\dagger}
\end{array}\right),
$$

satisfies canonical anticommutation relations $\left\{c_{\theta}, c_{\theta^{\prime}}^{\dagger}\right\}=\left\{d_{\theta}, d_{\theta^{\prime}}^{\dagger}\right\}=\delta_{\theta \theta^{\prime}}$ (the other anticommutators being equal to zero) and diagonalizes the transfer matrix $V_{\alpha}$ :

$$
V_{\alpha}=\left(\frac{c_{y}}{c_{x}^{*}}\right)^{N} \exp \left\{-\sum_{\theta \in \boldsymbol{\theta}_{\alpha}} \gamma_{\theta}\left(c_{\theta}^{\dagger} c_{\theta}+d_{\theta}^{\dagger} d_{\theta}-1\right)\right\} .
$$

Introduce the vacua $\alpha\langle v a c|$ and $|v a c\rangle_{\alpha}$ annihilated by all $\left\{c_{\theta}^{\dagger}\right\},\left\{d_{\theta}^{\dagger}\right\}$ and $\left\{c_{\theta}\right\},\left\{d_{\theta}\right\}$, respectively, and normalized as ${ }_{\alpha}\langle v a c \mid v a c\rangle_{\alpha}=1$. Left and right eigenvectors of $V_{\alpha}$ are then given by the multiparticle Fock states

$$
\begin{aligned}
{ }_{\alpha}\langle\boldsymbol{\theta} ; \boldsymbol{\phi}| & ={ }_{\alpha}\langle v a c| c_{\theta_{1}} \ldots c_{\theta_{m}} d_{\phi_{1}} \ldots d_{\phi_{n}}, \\
|\boldsymbol{\theta} ; \boldsymbol{\phi}\rangle_{\alpha} & =c_{\theta_{1}}^{\dagger} \ldots c_{\theta_{m}}^{\dagger} d_{\phi_{1}}^{\dagger} \ldots d_{\phi_{n}}^{\dagger}|v a c\rangle_{\alpha},
\end{aligned}
$$

and the corresponding eigenvalue is equal to $\left(\frac{c_{y}}{c_{x}^{*}}\right)^{N} \exp \left\{\sum_{\theta \in \boldsymbol{\theta}_{\alpha}} \gamma_{\theta}-\sum_{\theta \in \boldsymbol{\theta} \cup \boldsymbol{\phi}} \gamma_{\theta}\right\}$.

The states (14)-(15) simultaneously diagonalize the $U(1)$-charge and the translation operator,

$$
\begin{gathered}
Q=\sum_{\theta \in \boldsymbol{\theta}_{\alpha}}\left(c_{\theta}^{\dagger} c_{\theta}-d_{\theta}^{\dagger} d_{\theta}\right)=\sum_{\theta \in \boldsymbol{\theta}_{\alpha}}\left(a_{\theta}^{\dagger} a_{\theta}-b_{\theta}^{\dagger} b_{\theta}\right)=\sum_{y=0}^{N-1}\left(a_{y}^{\dagger} a_{y}-b_{y}^{\dagger} b_{y}\right), \\
T_{\alpha}=\exp \sum_{\theta \in \boldsymbol{\theta}_{\alpha}} i \theta\left(-c_{\theta}^{\dagger} c_{\theta}+d_{\theta}^{\dagger} d_{\theta}\right)=: \exp \sum_{y=0}^{N-1}\left(a_{y}^{\dagger} a_{y+1}-a_{y}^{\dagger} a_{y}+b_{y}^{\dagger} b_{y+1}-b_{y}^{\dagger} b_{y}\right): .
\end{gathered}
$$


The latter satisfies, e. g.,

$$
T_{\alpha}\left(\begin{array}{c}
a_{y}^{\dagger} \\
b_{y}
\end{array}\right) T_{\alpha}^{-1}=\left(\begin{array}{c}
a_{y-1}^{\dagger} \\
b_{y-1}
\end{array}\right), \quad T_{\alpha}\left(\begin{array}{c}
a_{y} \\
b_{y}^{\dagger}
\end{array}\right) T_{\alpha}^{-1}=\left(\begin{array}{c}
a_{y-1} \\
b_{y-1}^{\dagger}
\end{array}\right)
$$

under boundary conditions (10).

It is useful to note that the twist field (11) is $U(1)$-neutral and coincides with the identity operator twisted by translations of different periodicity:

$$
\mathcal{O}_{\alpha, \alpha^{\prime}}\left(y^{*}\right)=\left[\mathcal{O}_{\alpha^{\prime}, \alpha}\left(y^{*}\right)\right]^{-1}=T_{\alpha}^{-y} T_{\alpha^{\prime}}^{y}
$$

This can be seen by comparing linear transformations of fermions induced by the left and right side of this relation, and using that their action leaves the vector $|v a c\rangle$ invariant. Form factors of $\mathcal{O}_{\alpha, \alpha^{\prime}}\left(y^{*}\right)$ are therefore given, up to simple multiplicative factors, by the scalar products of the Fock states (14)-(15):

$$
{ }_{\alpha}\left\langle\boldsymbol{\theta} ; \boldsymbol{\phi}\left|\mathcal{O}_{\alpha, \alpha^{\prime}}\left(y^{*}\right)\right| \boldsymbol{\theta}^{\prime} ; \boldsymbol{\phi}^{\prime}\right\rangle_{\alpha^{\prime}}=\exp i y\left(\sum_{\theta \in \boldsymbol{\theta} \cup \boldsymbol{\phi}^{\prime}} \theta-\sum_{\theta \in \boldsymbol{\phi} \cup \boldsymbol{\theta}^{\prime}} \theta\right){ }_{\alpha}\left\langle\boldsymbol{\theta} ; \boldsymbol{\phi} \mid \boldsymbol{\theta}^{\prime} ; \boldsymbol{\phi}^{\prime}\right\rangle_{\alpha^{\prime}},
$$

where $\boldsymbol{\theta}, \boldsymbol{\phi} \subset \boldsymbol{\theta}_{\alpha}$ and $\boldsymbol{\theta}^{\prime}, \boldsymbol{\phi}^{\prime} \subset \boldsymbol{\theta}_{\alpha^{\prime}}$. Such scalar products normalized by the product of the vacua will be denoted by

$$
\mathcal{F}_{\alpha, \alpha^{\prime}}\left(\boldsymbol{\theta} ; \boldsymbol{\phi} \mid \boldsymbol{\theta}^{\prime} ; \boldsymbol{\phi}^{\prime}\right)=\frac{\alpha\left\langle\boldsymbol{\theta} ; \boldsymbol{\phi}\left|\mathcal{O}_{\alpha, \alpha^{\prime}}\left(0^{*}\right)\right| \boldsymbol{\theta}^{\prime} ; \boldsymbol{\phi}^{\prime}\right\rangle_{\alpha^{\prime}}}{{ }_{\alpha}\left\langle v a c\left|\mathcal{O}_{\alpha, \alpha^{\prime}}\left(0^{*}\right)\right| v a c\right\rangle_{\alpha^{\prime}}} .
$$

\section{Form factors}

\subsection{General setting}

Let us first recall a few results from [11, 13, 28]. Consider two sets of $2 L$ fermionic creation-annihilation operators generating equivalent Fock representations in the same space. Denote the corresponding $2^{L} \times 2^{L}$ matrices by $\left\{\psi_{i}\right\},\left\{\psi_{i}^{\dagger}\right\}$ and $\left\{\varphi_{i}\right\},\left\{\varphi_{i}^{\dagger}\right\}$ with $i=1, \ldots, L$ and combine them into $L$-columns $\boldsymbol{\psi}, \boldsymbol{\psi}^{\dagger}, \boldsymbol{\varphi}, \boldsymbol{\varphi}^{\dagger}$. Suppose there exists a unitary operator $\sigma$ such that

$$
\sigma\left(\begin{array}{l}
\varphi^{\dagger} \\
\varphi
\end{array}\right) \sigma^{-1}=\left(\begin{array}{ll}
\mathrm{A} & \mathrm{B} \\
\mathrm{C} & \mathrm{D}
\end{array}\right)\left(\begin{array}{l}
\boldsymbol{\psi}^{\dagger} \\
\boldsymbol{\psi}
\end{array}\right)
$$

where $\mathbf{A}, \mathbf{B}, \mathbf{C}, \mathbf{D}$ are some $L \times L$ matrices. The unitarity of $\sigma$ and canonical anticommutation relations imply that $\mathbf{B}=\overline{\mathbf{C}}, \mathbf{A}=\overline{\mathbf{D}}$ and

$$
\mathrm{DC}^{T}+\mathrm{CD}^{T}=0, \quad \mathrm{DD}^{\dagger}+\mathrm{CC}^{\dagger}=\mathbf{1} .
$$

Suppose that $\mathbf{D}$ is invertible. Then, up to inessential phase factor related to the choice of the vacua, one has

$$
{ }_{\psi}\langle v a c|\sigma| v a c\rangle_{\varphi}=|\operatorname{det} \mathbf{D}|^{\frac{1}{2}} .
$$

General matrix elements of $\sigma$ between Fock states of different types can be expressed as

$$
{ }_{\psi}\left\langle v a c\left|\psi_{i_{1}} \ldots \psi_{i_{m}} \sigma \varphi_{j_{1}}^{\dagger} \ldots \varphi_{j_{n}}^{\dagger}\right| v a c\right\rangle_{\varphi}=|\operatorname{det} \mathbf{D}|^{\frac{1}{2}} \cdot \operatorname{Pf} R
$$


Form factors of twist fields in the lattice Dirac theory

$$
R=\left(\begin{array}{cc}
R_{I \times I} & R_{I \times J} \\
R_{J \times I} & R_{J \times J}
\end{array}\right)
$$

where the entries of the blocks of $(m+n) \times(m+n)$ skew-symmetric matrix $R$ are given by the normalized two-particle form factors

$$
\begin{aligned}
& \left(R_{I \times I}\right)_{k l}=\left(\mathbf{D}^{-1} \mathbf{C}\right)_{i_{k} i_{l}}, \quad k, l=1, \ldots, m, \\
& \left(R_{I \times J}\right)_{k l}=-\left(R_{J \times I}\right)_{l k}=\mathbf{D}_{i_{k} j_{l}}^{-1}, \quad k=1, \ldots, m, \quad l=1, \ldots, n \text {, } \\
& \left(R_{J \times J}\right)_{k l}=\left(\overline{\mathbf{C D}}^{-1}\right)_{j_{k} j_{l}}, \quad k, l=1, \ldots, n .
\end{aligned}
$$

In the case of interest here, $\sigma$ is the identity operator and $L=2 N$. The creation-annihilation operators in each set are labeled by their $U(1)$-charges and the corresponding momenta. Thus e. g. $\boldsymbol{\psi}$ and $\boldsymbol{\varphi}$ are given by the $2 N$-columns $\left(\begin{array}{l}\mathbf{c} \\ \mathbf{d}\end{array}\right)$ built from the operators $c_{\theta}, d_{\theta}$ with $\theta \in \boldsymbol{\theta}_{\alpha}$ and $\theta \in \boldsymbol{\theta}_{\alpha^{\prime}}$, respectively. $\mathbf{A}, \mathbf{B}, \mathbf{C}$, D can therefore be seen as block $2 \times 2$ matrices with block entries indexed by $\theta \in \boldsymbol{\theta}_{\alpha^{\prime}}, \theta^{\prime} \in \boldsymbol{\theta}_{\alpha}$. To find their explicit form, note that for $\theta \in \boldsymbol{\theta}_{\alpha^{\prime}}$ one has

$$
\begin{aligned}
& \left(\begin{array}{c}
c_{\theta}^{\dagger} \\
d_{\theta}
\end{array}\right)=\frac{1}{N} \sum_{\theta^{\prime} \in \boldsymbol{\theta}_{\alpha}} \frac{1-e^{-2 \pi i \nu}}{1-e^{i\left(\theta^{\prime}-\theta\right)}} U^{\dagger}(\theta) U\left(\theta^{\prime}\right)\left(\begin{array}{c}
c_{\theta^{\prime}}^{\dagger} \\
d_{\theta^{\prime}}
\end{array}\right), \\
& \left(\begin{array}{c}
c_{\theta} \\
d_{\theta}^{\dagger}
\end{array}\right)=\frac{1}{N} \sum_{\theta^{\prime} \in \boldsymbol{\theta}_{\alpha}} \frac{1-e^{2 \pi i \nu}}{1-e^{i\left(\theta-\theta^{\prime}\right)}} U^{T}(\theta) \bar{U}\left(\theta^{\prime}\right)\left(\begin{array}{c}
c_{\theta^{\prime}} \\
d_{\theta^{\prime}}^{\dagger}
\end{array}\right),
\end{aligned}
$$

where $U(\theta)$ is defined by (12). Therefore, introducing the notation

$$
\begin{array}{ll}
\Lambda_{\theta, \theta^{\prime}}=e^{i(\pi \nu+\theta) / 2} \delta_{\theta, \theta^{\prime}}, & \theta, \theta^{\prime} \in \boldsymbol{\theta}_{\alpha}, \\
\Lambda_{\theta, \theta^{\prime}}^{\prime}=e^{i(\pi \nu-\theta) / 2} \delta_{\theta, \theta^{\prime}}, & \theta, \theta^{\prime} \in \boldsymbol{\theta}_{\alpha^{\prime}}, \\
C_{\theta, \theta^{\prime}}=\frac{\sin \pi \nu}{N} \frac{f_{1}(\theta) f_{2}\left(\theta^{\prime}\right)-f_{2}(\theta) f_{1}\left(\theta^{\prime}\right)}{\sin \frac{\theta^{\prime}-\theta}{2}}, & \theta \in \boldsymbol{\theta}_{\alpha^{\prime}}, \theta^{\prime} \in \boldsymbol{\theta}_{\alpha}, \\
D_{\theta, \theta^{\prime}}=\frac{\sin \pi \nu}{N} \frac{f_{1}(\theta) \bar{f}_{1}\left(\theta^{\prime}\right)+f_{2}(\theta) \bar{f}_{2}\left(\theta^{\prime}\right)}{\sin \frac{\theta^{\prime}-\theta}{2}}, & \theta \in \boldsymbol{\theta}_{\alpha^{\prime}}, \theta^{\prime} \in \boldsymbol{\theta}_{\alpha},
\end{array}
$$

we find that

$$
\mathbf{C}=\left(\begin{array}{cc}
0 & \Lambda^{\prime} C \Lambda \\
-\bar{\Lambda}^{\prime} C \bar{\Lambda} & 0
\end{array}\right), \quad \mathbf{D}=\left(\begin{array}{cc}
-\Lambda^{\prime} D \Lambda & 0 \\
0 & -\bar{\Lambda}^{\prime} D \bar{\Lambda}
\end{array}\right)
$$

This in turn implies that the vacuum expectation value of twist field and its nonzero two-particle form factors are given by

$$
\begin{aligned}
& { }_{\alpha}\left\langle\text { vac }\left|\mathcal{O}_{\alpha, \alpha^{\prime}}\left(0^{*}\right)\right| v a c\right\rangle_{\alpha^{\prime}}=|\operatorname{det} D|, \\
& \mathcal{F}_{\alpha, \alpha^{\prime}}\left(\theta ; \mid \theta^{\prime} ;\right)=-e^{i\left(\theta^{\prime}-\theta-2 \pi \nu\right) / 2} D_{\theta, \theta^{\prime}}^{-1}, \\
& \mathcal{F}_{\alpha, \alpha^{\prime}}\left(; \theta \mid ; \theta^{\prime}\right)=-e^{i\left(\theta-\theta^{\prime}+2 \pi \nu\right) / 2} D_{\theta, \theta^{\prime}}^{-1}, \\
& \mathcal{F}_{\alpha, \alpha^{\prime}}\left(\theta ; \theta^{\prime} \mid ;\right)=-e^{i\left(\theta^{\prime}-\theta\right) / 2}\left(D^{-1} C\right)_{\theta, \theta^{\prime}}, \\
& \mathcal{F}_{\alpha, \alpha^{\prime}}\left(; \mid \theta ; \theta^{\prime}\right)=-e^{i\left(\theta-\theta^{\prime}\right) / 2}\left(\bar{C} D^{-1}\right)_{\theta, \theta^{\prime}} .
\end{aligned}
$$


One also has

$$
\mathcal{F}_{\alpha, \alpha^{\prime}}\left(; \mid \theta ; \theta^{\prime}\right)=-\overline{\mathcal{F}_{\alpha^{\prime}, \alpha}\left(\theta ; \theta^{\prime} \mid ;\right)}
$$

although this is not immediately obvious from (28)-(29). This reduces our task to the computation of determinant and inverse of $D$ and of the product $D^{-1} C$.

The relations (23) -(24) imply that the elements of $C$ and $D$ remain invariant if one multiplies $U(\theta)$ from the left by a unitary matrix independent of $\theta$. Together with (12), this gives

$$
\begin{aligned}
C_{\theta, \theta^{\prime}} & =\frac{\sin \pi \nu}{2 N} \frac{\chi_{\theta} \chi_{-\theta^{\prime}}-\chi_{-\theta} \chi_{\theta^{\prime}}}{\sin \frac{\theta^{\prime}-\theta}{2}}, \\
D_{\theta, \theta^{\prime}} & =\frac{\sin \pi \nu}{2 N} \frac{\chi_{\theta} \chi_{-\theta^{\prime}}+\chi_{-\theta} \chi_{\theta^{\prime}}}{\sin \frac{\theta^{\prime}-\theta}{2}} .
\end{aligned}
$$

The matrices $C$ and $D$ are therefore very simply related to the ones appearing in the Ising model theory, see Lemma 3.3 in [13]; the main and almost only difference between the two cases is the change in the spectrum of quasimomenta. We will now follow [13] to establish elliptic representations for $C$ and $D$, which then will be used to calculate $\operatorname{det} D, D^{-1}$ and $D^{-1} C$.

\subsection{Elliptic parametrization}

Spectral curve (3) is a torus which can be conveniently uniformized by the Jacobi elliptic functions of modulus $k=\frac{\sinh 2 \mathcal{K}_{x}^{*}}{\sinh 2 \mathcal{K}_{y}}$. Let us denote $K=\mathbf{K}(k), K^{\prime}=\mathbf{K}\left(\sqrt{1-k^{2}}\right)$, where $\mathbf{K}(k)$ stands for the complete elliptic integral of the first kind. Then [13, 24] the functions

$$
\begin{aligned}
z(u) & =\frac{\operatorname{sn}(u+i \eta)}{\operatorname{sn}(u-i \eta)}, \\
\lambda(u) & =[k \operatorname{sn}(u+i \eta) \operatorname{sn}(u-i \eta)]^{-1},
\end{aligned}
$$

with $\eta \in\left(-\frac{K^{\prime}}{2}, 0\right)$ determined by $\sinh 2 \mathcal{K}_{x}=i \operatorname{sn} 2 i \eta$, satisfy the relation (33) written in the form

$$
s_{x}\left(\lambda+\lambda^{-1}\right)+s_{y}\left(z+z^{-1}\right)=2 c_{x} c_{y} .
$$

The formulas (33)-(34) bijectively map the real interval $\mathcal{C}_{u}=\{u \mid \operatorname{Re} u \in$ $[-K, K), \operatorname{Im} u=0\}$ to $\mathcal{C}_{\theta}=\left\{(z, \lambda)=\left(e^{i \theta}, e^{\gamma_{\theta}}\right) \mid \theta \in[0,2 \pi)\right\}$. The inverse image of the point $\left(e^{i \theta}, e^{\gamma_{\theta}}\right) \in \mathcal{C}_{\theta}$ in $\mathcal{C}_{u}$ will be denoted by $u_{\theta}$. Note that for $\theta \in(0, \pi]$ one has $u_{\theta}=-u_{2 \pi-\theta}$. It is also useful to define the function $x_{\theta}=\frac{\pi u_{\theta}}{2 K}$, which continuously increases from $-\frac{\pi}{2}$ to $\frac{\pi}{2}$ when $\theta$ varies from 0 to $2 \pi$.

Lemma 4.1 in [13] shows that under the above parametrization matrix elements (31) - (32) can be written as

$$
\begin{aligned}
C_{\theta, \theta^{\prime}} & =\frac{-i s_{x}^{*} \sin \pi \nu}{N \sqrt{\sinh \gamma_{\theta} \sinh \gamma_{\theta^{\prime}}}} \operatorname{cn}\left(u_{\theta}+u_{\theta^{\prime}}\right), \\
D_{\theta, \theta^{\prime}} & =\frac{-s_{y} \sin \pi \nu}{N \sqrt{\sinh \gamma_{\theta} \sinh \gamma_{\theta^{\prime}}}} \frac{\operatorname{dn}\left(u_{\theta}-u_{\theta^{\prime}}\right)}{\operatorname{sn}\left(u_{\theta}-u_{\theta^{\prime}}\right)} .
\end{aligned}
$$


Form factors of twist fields in the lattice Dirac theory

We will also need Jacobi theta functions $\vartheta_{1 \ldots 4}(z)$ of nome $q=e^{i \pi \tau}$, which are related to the elliptic modulus and half-periods by

$$
k=\frac{\vartheta_{2}^{2}}{\vartheta_{3}^{2}}, \quad 2 K=\pi \vartheta_{3}^{2}, \quad 2 i K^{\prime}=\pi \tau \vartheta_{3}^{2},
$$

where $\vartheta_{i}=\vartheta_{i}(0)$ for $i=2,3,4$. The quantities $\operatorname{det} D$ and $D^{-1}$ can be computed in terms of these functions using Frobenius determinant identity, while $D^{-1} C$ can be found from a theta functional analog of the Lagrange interpolation formula (see Section 5 of [13] for the details of a similar cumbersome calculation). The result is as follows:

$$
\begin{aligned}
& \operatorname{det} D=\frac{\vartheta_{3}\left(X_{\alpha^{\prime}}-X_{\alpha}\right)}{\vartheta_{3}}\left(\frac{s_{y} \sin \pi \nu}{N} \frac{\vartheta_{2} \vartheta_{4}}{\vartheta_{3}}\right)^{N}(-1)^{N}\left[\prod_{\theta \in \boldsymbol{\theta}_{\alpha}} \sinh \gamma_{\theta} \prod_{\theta \in \boldsymbol{\theta}_{\alpha^{\prime}}} \sinh \gamma_{\theta}\right]^{-\frac{1}{2}} \times \\
& \times \frac{\prod_{\theta, \theta^{\prime} \in \boldsymbol{\theta}_{\alpha}, \theta<\theta^{\prime}} \vartheta_{1}\left(x_{\theta^{\prime}}-x_{\theta}\right) \prod_{\theta, \theta^{\prime} \in \boldsymbol{\theta}_{\alpha^{\prime}}, \theta<\theta^{\prime}} \vartheta_{1}\left(x_{\theta}-x_{\theta^{\prime}}\right)}{\prod_{\theta \in \boldsymbol{\theta}_{\alpha}, \theta^{\prime} \in \boldsymbol{\theta}_{\alpha^{\prime}}} \vartheta_{1}\left(x_{\theta^{\prime}}-x_{\theta}\right)}, \\
& D_{\theta, \theta^{\prime}}^{-1}=\frac{\vartheta_{3}\left(X_{\alpha^{\prime}}-X_{\alpha}+x_{\theta}-x_{\theta^{\prime}}\right)}{\vartheta_{3}\left(X_{\alpha^{\prime}}-X_{\alpha}\right) \vartheta_{1}\left(x_{\theta^{\prime}}-x_{\theta}\right)}\left(\frac{s_{y} \sin \pi \nu}{N} \frac{\vartheta_{2} \vartheta_{4}}{\vartheta_{3}}\right)^{-1}\left[\sinh \gamma_{\theta} \sinh \gamma_{\theta^{\prime}}\right]^{\frac{1}{2}} \times \\
& \times \frac{\prod_{\theta^{\prime \prime} \in \boldsymbol{\theta}_{\alpha^{\prime}}} \vartheta_{1}\left(x_{\theta}-x_{\theta^{\prime \prime}}\right) \prod_{\theta^{\prime \prime} \in \boldsymbol{\theta}_{\alpha}} \vartheta_{1}\left(x_{\theta^{\prime}}-x_{\theta^{\prime \prime}}\right)}{\prod_{\theta^{\prime \prime} \in \boldsymbol{\theta}_{\alpha}, \theta^{\prime \prime} \neq \theta} \vartheta_{1}\left(x_{\theta}-x_{\theta^{\prime \prime}}\right) \prod_{\theta^{\prime \prime} \in \boldsymbol{\theta}_{\alpha^{\prime}}, \theta^{\prime \prime} \neq \theta^{\prime}} \vartheta_{1}\left(x_{\theta^{\prime}}-x_{\theta^{\prime \prime}}\right)}, \\
&\left(D^{-1} C\right)_{\theta, \theta^{\prime}}=-i \frac{\vartheta_{2}\left(X_{\alpha^{\prime}}-X_{\alpha}+x_{\theta}+x_{\theta^{\prime}}\right)}{\vartheta_{3}\left(X_{\alpha^{\prime}}-X_{\alpha}\right)}\left[\frac{\sinh \gamma_{\theta}}{\sinh \gamma_{\theta^{\prime}}}\right]^{\frac{1}{2}} \times \\
& \times \frac{\prod_{\theta^{\prime \prime} \in \boldsymbol{\theta}_{\alpha^{\prime}}} \vartheta_{1}\left(x_{\theta}-x_{\theta^{\prime \prime}}\right) \prod_{\theta^{\prime \prime} \in \boldsymbol{\theta}_{\alpha}, \theta^{\prime \prime} \neq \theta} \vartheta_{4}\left(x_{\theta^{\prime}}+x_{\theta^{\prime \prime}}\right)}{\prod_{\theta^{\prime \prime} \in \boldsymbol{\theta}_{\alpha^{\prime}}} \vartheta_{4}\left(x_{\theta^{\prime}}+x_{\theta^{\prime \prime}}\right) \prod_{\theta^{\prime \prime} \in \boldsymbol{\theta}_{\alpha}, \theta^{\prime \prime} \neq \theta} \vartheta_{1}\left(x_{\theta}-x_{\theta^{\prime \prime}}\right)},
\end{aligned}
$$

with $X_{\alpha}=\sum_{\theta \in \boldsymbol{\theta}_{\alpha}} x_{\theta}$. In the next subsection, the answer (37)-(39) is rewritten in a somewhat different form, which turns out to be more suitable for the analysis of the thermodynamic limit and for the computation of multiparticle form factors.

\subsection{VEV and form factors}

We illustrate the procedure by transforming the expression for $\left(D^{-1} C\right)_{\theta, \theta^{\prime}}$. First rewrite the second line of (39) as

$$
\frac{\vartheta_{2} \vartheta_{4}}{\vartheta_{3}} \frac{G_{\theta}^{-}}{G_{\theta^{\prime}}^{+} \vartheta_{4}\left(x_{\theta}+x_{\theta^{\prime}}\right)} \frac{\prod_{\theta^{\prime \prime} \in \boldsymbol{\theta}_{\alpha^{\prime}}} \operatorname{sn}\left(u_{\theta}-u_{\theta^{\prime \prime}}\right)}{\prod_{\theta^{\prime \prime} \in \boldsymbol{\theta}_{\alpha}, \theta^{\prime \prime} \neq \theta} \operatorname{sn}\left(u_{\theta}-u_{\theta^{\prime \prime}}\right)},
$$

where the functions $G_{\theta}^{ \pm}$are defined by

$$
G_{\theta}^{ \pm}=G_{2 \pi-\theta}^{\mp}=\frac{\prod_{\theta^{\prime \prime} \in \boldsymbol{\theta}_{\alpha^{\prime}}} \vartheta_{4}\left(x_{\theta} \pm x_{\theta^{\prime \prime}}\right)}{\prod_{\theta^{\prime \prime} \in \boldsymbol{\theta}_{\alpha}} \vartheta_{4}\left(x_{\theta} \pm x_{\theta^{\prime \prime}}\right)}
$$

The last factor in (40) can be rewritten using the relation $\operatorname{sn}\left(u_{\theta}-u_{\theta^{\prime}}\right)=\frac{s_{y} \sin \frac{\theta-\theta^{\prime}}{2}}{\sinh \frac{\gamma_{\theta}+\gamma_{\theta^{\prime}}}{2}}$, see e. g. formula (4.5) in [13]. The resulting products of sine functions can be calculated explicitly: for $\theta \in \boldsymbol{\theta}_{\alpha}$, one has

$$
2^{N-1} \prod_{\theta^{\prime \prime} \in \boldsymbol{\theta}_{\alpha^{\prime}}} \sin \frac{\theta-\theta^{\prime \prime}}{2}=(-1)^{\frac{N \theta}{2 \pi}-\alpha+N} \sin \pi \nu
$$


Form factors of twist fields in the lattice Dirac theory

$$
2^{N-1} \prod_{\theta^{\prime \prime} \in \boldsymbol{\theta}_{\alpha}, \theta^{\prime \prime} \neq \theta} \sin \frac{\theta-\theta^{\prime \prime}}{2}=(-1)^{\frac{N \theta}{2 \pi}-\alpha+N-1} N .
$$

The remaining products of sinh's can be combined into the function

$$
H_{\theta}=\frac{\prod_{\theta^{\prime \prime} \in \boldsymbol{\theta}_{\alpha^{\prime}}} \sinh \frac{\gamma_{\theta}+\gamma_{\theta^{\prime \prime}}}{2}}{\prod_{\theta^{\prime \prime} \in \boldsymbol{\theta}_{\alpha}} \sinh \frac{\gamma_{\theta}+\gamma_{\theta^{\prime \prime}}}{2}} .
$$

There exists a simple combination of $G_{\theta}^{ \pm}$and $H_{\theta}$ independent of $\theta$, namely

$$
\frac{G_{\theta}^{+} G_{\theta}^{-}}{H_{\theta}}=\frac{G_{\pi}^{2}}{H_{\pi}}
$$

with $G_{\pi}=G_{\pi}^{ \pm}$. The identity (41) can be proven using the standard addition formulas for theta functions, their relation to the elliptic functions and the formula (4.8) in [13], relating elliptic and trigonometric parametrization.

Summarizing the above transformations and using (41), we can rewrite the formula (39) in the following equivalent form:

$$
\left(D^{-1} C\right)_{\theta, \theta^{\prime}}=\frac{i s_{y} \sin \pi \nu e^{\left(\eta_{\theta}+\eta_{\theta^{\prime}}\right) / 2}}{N \sqrt{\sinh \gamma_{\theta} \sinh \gamma_{\theta^{\prime}}}} \frac{\vartheta_{2} \vartheta_{4}}{\vartheta_{3}} \frac{\vartheta_{2}\left(X_{\alpha^{\prime}}-X_{\alpha}+x_{\theta}+x_{\theta^{\prime}}\right)}{\vartheta_{3}\left(X_{\alpha^{\prime}}-X_{\alpha}\right) \vartheta_{4}\left(x_{\theta}+x_{\theta^{\prime}}\right)},
$$

where the function $\eta_{\theta}$ is given by

$$
\eta_{\theta}=2 \ln \frac{G_{\pi}}{G_{\theta}^{+}}-\ln H_{\pi}
$$

Analogous manipulations with (37)-(38) lead to the representations

$$
\begin{aligned}
& \operatorname{det} D=(-1)^{N} \frac{\vartheta_{3}\left(X_{\alpha^{\prime}}-X_{\alpha}\right)}{\vartheta_{3}} \prod_{\theta \in \boldsymbol{\theta}_{\alpha}} e^{-\eta_{\theta} / 4} \prod_{\theta \in \boldsymbol{\theta}_{\alpha^{\prime}}} e^{\eta_{\theta} / 4}, \\
& D_{\theta, \theta^{\prime}}^{-1}=\frac{s_{y} \sin \pi \nu e^{\left(\eta_{\theta}-\eta_{\theta^{\prime}}\right) / 2}}{N \sqrt{\sinh \gamma_{\theta} \sinh \gamma_{\theta^{\prime}}}} \frac{\vartheta_{2} \vartheta_{4}}{\vartheta_{3}} \frac{\vartheta_{3}\left(X_{\alpha^{\prime}}-X_{\alpha}+x_{\theta}-x_{\theta^{\prime}}\right)}{\vartheta_{3}\left(X_{\alpha^{\prime}}-X_{\alpha}\right) \vartheta_{1}\left(x_{\theta}-x_{\theta^{\prime}}\right)} .
\end{aligned}
$$

By (25), the formula (44) gives the vacuum expectation value of twist field. Non-zero crossed and non-crossed two-particle form factors are determined by (26), (27), (45) and (28), (301), (42), respectively.

Multiparticle form factors (16) can be obtained from (18)-(22). They do not vanish only if the charge preserving condition $m-m^{\prime}=n-n^{\prime}$ is satisfied, where $m=\#(\boldsymbol{\theta})$, $n=\#(\phi), m^{\prime}=\#\left(\boldsymbol{\theta}^{\prime}\right)$ and $n^{\prime}=\#\left(\boldsymbol{\phi}^{\prime}\right)$. Under this restriction, one has

$$
\begin{gathered}
\mathcal{F}_{\alpha, \alpha^{\prime}}\left(\boldsymbol{\theta}, \boldsymbol{\phi} \mid \boldsymbol{\theta}^{\prime}, \boldsymbol{\phi}^{\prime}\right)=\left(\frac{\vartheta_{2} \vartheta_{4}}{\vartheta_{3}} s_{y} \sin \pi \nu\right)^{m+n^{\prime}} \prod_{\theta \in \boldsymbol{\theta}} \frac{e^{-i(\theta+\pi \nu) / 2+\eta_{\theta} / 2}}{\sqrt{N \sinh \gamma_{\theta}}} \prod_{\phi \in \boldsymbol{\phi}} \frac{e^{i(\phi+\pi \nu) / 2+\eta_{\phi} / 2}}{\sqrt{N \sinh \gamma_{\phi}}} \times \\
\times(-1)^{\frac{\left(m^{\prime}+n\right)\left(m-n^{\prime}-1\right)}{2}} \prod_{\theta^{\prime} \in \boldsymbol{\theta}^{\prime}} \frac{e^{i\left(\theta^{\prime}-\pi \nu\right) / 2-\eta_{\theta^{\prime}} / 2}}{\sqrt{N \sinh \gamma_{\theta^{\prime}}}} \prod_{\phi^{\prime} \in \phi^{\prime}} \frac{e^{-i\left(\phi^{\prime}-\pi \nu\right) / 2-\eta_{\phi^{\prime}} / 2}}{\sqrt{N \sinh \gamma_{\phi^{\prime}}}} \cdot \operatorname{det} \mathcal{R},
\end{gathered}
$$

where the $\left(m+n^{\prime}\right) \times\left(m+n^{\prime}\right)$ matrix $\mathcal{R}$ has $2 \times 2$ block form, $\mathcal{R}=\left(\begin{array}{cc}\mathcal{A} & \mathcal{B} \\ \mathcal{C} & \mathcal{D}\end{array}\right)$, and is explicitly given by

$$
\mathcal{A}_{j k}=-\frac{i \vartheta_{2}\left(X_{\alpha^{\prime}}-X_{\alpha}+x_{\theta_{j}}+x_{\phi_{k}}\right)}{\vartheta_{3}\left(X_{\alpha^{\prime}}-X_{\alpha}\right) \vartheta_{4}\left(x_{\theta_{j}}+x_{\phi_{k}}\right)}, \quad j=1, \ldots, m, \quad k=1, \ldots, n,
$$


Form factors of twist fields in the lattice Dirac theory

$$
\begin{aligned}
& \mathcal{B}_{j k}=-\frac{\vartheta_{3}\left(X_{\alpha^{\prime}}-X_{\alpha}+x_{\theta_{j}}-x_{\theta_{k}^{\prime}}\right)}{\vartheta_{3}\left(X_{\alpha^{\prime}}-X_{\alpha}\right) \vartheta_{1}\left(x_{\theta_{j}}-x_{\theta_{k}^{\prime}}\right)}, j=1, \ldots, m, \quad k=1, \ldots, m^{\prime}, \\
& \mathcal{C}_{j k}=-\frac{\vartheta_{3}\left(X_{\alpha^{\prime}}-X_{\alpha}+x_{\phi_{k}}-x_{\phi_{j}^{\prime}}\right)}{\vartheta_{3}\left(X_{\alpha^{\prime}}-X_{\alpha}\right) \vartheta_{1}\left(x_{\phi_{k}}-x_{\phi_{j}^{\prime}}\right)}, \quad j=1, \ldots, n^{\prime}, \quad k=1, \ldots, n, \\
& \mathcal{D}_{j k}=-\frac{i \vartheta_{2}\left(X_{\alpha}-X_{\alpha^{\prime}}+x_{\theta_{k}^{\prime}}+x_{\phi_{j}^{\prime}}\right)}{\vartheta_{3}\left(X_{\alpha}-X_{\alpha^{\prime}}\right) \vartheta_{4}\left(x_{\theta_{k}^{\prime}}+x_{\phi_{j}^{\prime}}\right)}, \quad j=1, \ldots, n^{\prime}, \quad k=1, \ldots, m^{\prime} .
\end{aligned}
$$

The determinant of $\mathcal{R}$ in (46) can be evaluated in a closed form using Frobenius identity. Given $2 L$ indeterminates $z_{1}, \ldots, z_{L}$ and $z_{1}^{\prime}, \ldots, z_{L}^{\prime}$, it expresses the determinant of the elliptic Cauchy matrix $\Omega$ with elements

$$
\Omega_{j k}=\frac{\vartheta_{1}\left(z_{j}-z_{k}^{\prime}+r\right)}{\vartheta_{1}(r) \vartheta_{1}\left(z_{j}-z_{k}^{\prime}\right)}, \quad j, k=1, \ldots, L, \quad r \in \mathbb{C},
$$

in the product form

$$
\operatorname{det} \Omega=\frac{\vartheta_{1}\left(\sum_{j}^{L} z_{j}-\sum_{j}^{L} z_{j}^{\prime}+r\right)}{\vartheta_{1}(r)} \frac{\prod_{j<k}^{L} \vartheta_{1}\left(z_{j}-z_{k}\right) \vartheta_{1}\left(z_{k}^{\prime}-z_{j}^{\prime}\right)}{\prod_{j, k}^{L} \vartheta_{1}\left(z_{j}-z_{k}^{\prime}\right)} .
$$

Setting $L=m+n^{\prime}, r=X_{\alpha^{\prime}}-X_{\alpha}+\frac{\pi}{2}-\frac{\pi \tau}{2}$ and

$$
\begin{aligned}
& z_{j}= \begin{cases}x_{\theta_{j}}+\frac{\pi \tau}{2} & \text { for } j=1, \ldots, m, \\
-x_{\phi_{j-m}^{\prime}} & \text { for } j=m+1, \ldots, m+n^{\prime},\end{cases} \\
& z_{j}^{\prime}= \begin{cases}-x_{\phi_{j}} & \text { for } j=1, \ldots, n, \\
x_{\theta_{j-n}^{\prime}}+\frac{\pi \tau}{2} & \text { for } j=n+1, \ldots, n+m^{\prime},\end{cases}
\end{aligned}
$$

one can check that $\Omega$ coincides with $\mathcal{R}$ up to diagonal matrix factors. It then follows from (48) that

$$
\begin{gathered}
\operatorname{det} \mathcal{R}=(-1)^{m^{\prime}\left(n^{\prime}+1\right)} i^{-\left(m-m^{\prime}\right)^{2}} \frac{\vartheta_{p}\left(X_{\alpha^{\prime}}-X_{\alpha}+\sum_{\theta \in \boldsymbol{\theta}} x_{\theta}-\sum_{\theta^{\prime} \in \boldsymbol{\theta}^{\prime}} x_{\theta^{\prime}}+\sum_{\phi \in \boldsymbol{\phi}} x_{\phi}-\sum_{\phi^{\prime} \in \boldsymbol{\phi}^{\prime}} x_{\phi^{\prime}}\right)}{\vartheta_{3}\left(X_{\alpha^{\prime}}-X_{\alpha}\right)} \times \\
\times \frac{\prod_{1 \leq j<k \leq m}\left(x_{\theta_{j}}-x_{\theta_{k}}\right) \prod_{1 \leq j<k \leq n} \vartheta_{1}\left(x_{\phi_{j}}-x_{\phi_{k}}\right) \prod_{1 \leq j<k \leq m^{\prime}} \vartheta_{1}\left(x_{\theta_{k}^{\prime}}-x_{\theta_{j}^{\prime}}\right) \prod_{1 \leq j<k \leq n^{\prime}} \vartheta_{1}\left(x_{\phi_{k}^{\prime}}-x_{\phi_{j}^{\prime}}\right)}{\prod_{j=1}^{m} \prod_{k=1}^{m^{\prime}} \vartheta_{1}\left(x_{\theta_{j}}-x_{\theta_{k}^{\prime}}\right) \prod_{j=1}^{n^{\prime}} \prod_{k=1}^{n} \vartheta_{1}\left(x_{\phi_{k}}-x_{\phi_{j}^{\prime}}\right)} \times \\
\times \frac{\prod_{j=1}^{m} \prod_{k=1}^{n^{\prime}} \vartheta_{4}\left(x_{\theta_{j}}+x_{\phi_{k}^{\prime}}\right) \prod_{j=1}^{n} \prod_{k=1}^{m^{\prime}} \vartheta_{4}\left(x_{\theta_{k}^{\prime}}+x_{\phi_{j}}\right)}{\prod_{j=1}^{m} \prod_{k=1}^{n} \vartheta_{4}\left(x_{\theta_{j}}+x_{\phi_{k}}\right) \prod_{j=1}^{n^{\prime}} \prod_{k=1}^{m^{\prime}} \vartheta_{4}\left(x_{\theta_{k}^{\prime}}+x_{\phi_{j}^{\prime}}\right)},
\end{gathered}
$$

where $p=2$ for $m-m^{\prime} \in 2 \mathbb{Z}+1$ and $p=3$ for $m-m^{\prime} \in 2 \mathbb{Z}$. Together with (46), this gives our main result - a completely explicit factorized formula for any multiparticle matrix element of twist field. 


\subsection{Thermodynamic limit}

Form factors found above simplify in the thermodynamic limit $N \rightarrow \infty$. To explain these simplifications, consider e. g. the expression (44) for the vacuum expectation value (25). We need to evaluate the asymptotics of (i) $X_{\alpha^{\prime}}-X_{\alpha}$ and (ii) $\eta_{\theta}$. Both quantities can be written in the same form

$$
\sum_{\theta^{\prime} \in \boldsymbol{\theta}_{\alpha^{\prime}}} f_{\theta^{\prime}}-\sum_{\theta^{\prime} \in \boldsymbol{\theta}_{\alpha}} f_{\theta^{\prime}}
$$

where the function $f_{\theta^{\prime}}$ is defined for $\theta^{\prime} \in[0,2 \pi]$. In the case (i) one has $f_{\theta^{\prime}}=x_{\theta^{\prime}}$, while in the case (ii) $f_{\theta^{\prime}}$ is given by

$$
f_{\theta^{\prime}}=2 \ln \frac{\vartheta_{4}\left(x_{\theta^{\prime}}\right)}{\vartheta_{4}\left(x_{\theta}+x_{\theta^{\prime}}\right)}-\ln \sinh \frac{\gamma_{\pi}+\gamma_{\theta^{\prime}}}{2} .
$$

The most important distinction between the two situations is that in the first case one has $f_{2 \pi}-f_{0}=\pi$, whereas (51) extends to a continuous $2 \pi$-periodic function on the real line.

As $N \rightarrow \infty$, one has the estimate

$$
f_{\frac{2 \pi}{N}\left(k+\alpha^{\prime}\right)}-f_{\frac{2 \pi}{N}(k+\alpha)} \sim \frac{2 \pi \nu}{N} f_{\frac{2 \pi k}{N}}^{\prime}
$$

with $k=0, \ldots, N-1$. The sum $\frac{2 \pi}{N} \sum_{k=0}^{N-1} f_{\frac{2 \pi k}{N}}^{\prime}$ transforms into the integral $\int_{0}^{2 \pi} f_{\theta}^{\prime} d \theta$, which yields

$$
\lim _{N \rightarrow \infty}\left(X_{\alpha^{\prime}}-X_{\alpha}\right)=\pi \nu, \quad \lim _{N \rightarrow \infty} \eta_{\theta}=0 .
$$

In the same way one shows that

$$
\lim _{N \rightarrow \infty}\left(\sum_{\theta \in \boldsymbol{\theta}_{\alpha^{\prime}}} \eta_{\theta}-\sum_{\theta \in \boldsymbol{\theta}_{\alpha}} \eta_{\theta}\right)=0 .
$$

Rigorous mathematical proofs of (52)-( using a kind of Sommerfeld-Watson transform of the sums (50).

It then follows from (25), (44) that the thermodynamic limit of the vacuum expectation value of twist field is given by the simple expression

$$
\left\langle\mathcal{O}_{\nu}\right\rangle \stackrel{\text { def }}{=} \lim _{N \rightarrow \infty} \alpha\left\langle v a c\left|\mathcal{O}_{\alpha, \alpha^{\prime}}\left(0^{*}\right)\right| v a c\right\rangle_{\alpha^{\prime}}=\frac{\vartheta_{3}(\pi \nu \mid \tau)}{\vartheta_{3}(0 \mid \tau)}
$$

where the arguments of theta functions and the half-period ratio are now indicated explicitly for further convenience. As one could expect on general grounds, the r.h.s. of (54) depends on $\alpha, \alpha^{\prime}$ only via the difference $\nu=\alpha^{\prime}-\alpha$. Note that this relation has the same form as the first formula on p. 187 of [25].

Particle quasimomenta in the thermodynamic limit uniformly fill the interval $[0,2 \pi]$. Summation over each of them in the form factor expansions of correlation functions transforms into integration: $\frac{1}{N} \sum_{\theta} \rightarrow \frac{1}{2 \pi} \int_{0}^{2 \pi} d \theta$. Although this naive procedure is plagued by the appearance of annihilation poles in the crossed form factors, it suggests to consider

$$
\mathbf{F}_{\nu}\left(\boldsymbol{\theta} ; \boldsymbol{\phi} \mid \boldsymbol{\theta}^{\prime} ; \boldsymbol{\phi}^{\prime}\right) \stackrel{\text { def }}{=} \lim _{N \rightarrow \infty} N^{\frac{m+n+m^{\prime}+n^{\prime}}{2}} \mathcal{F}_{\alpha, \alpha^{\prime}}\left(\boldsymbol{\theta} ; \boldsymbol{\phi} \mid \boldsymbol{\theta}^{\prime} ; \boldsymbol{\phi}^{\prime}\right)
$$


The asymptotics (52) implies that the limiting two-particle form factors are given by

$$
\begin{aligned}
& \mathbf{F}_{\nu}\left(\theta ; \mid \theta^{\prime} ;\right)=\overline{\mathbf{F}_{\nu}\left(; \theta \mid ; \theta^{\prime}\right)}= \\
& =-\frac{s_{y} \sin \pi \nu e^{i\left(\theta^{\prime}-\theta-2 \pi \nu\right) / 2}}{\sqrt{\sinh \gamma_{\theta} \sinh \gamma_{\theta^{\prime}}}} \frac{\vartheta_{2}(0 \mid \tau) \vartheta_{4}(0 \mid \tau)}{\vartheta_{3}(0 \mid \tau) \vartheta_{3}(\pi \nu \mid \tau)} \frac{\vartheta_{3}\left(x_{\theta}-x_{\theta^{\prime}}+\pi \nu \mid \tau\right)}{\vartheta_{1}\left(x_{\theta}-x_{\theta^{\prime}} \mid \tau\right)}, \\
& \mathbf{F}_{\nu}\left(\theta ; \theta^{\prime} \mid ;\right)=-\overline{\mathbf{F}_{-\nu}\left(; \mid \theta ; \theta^{\prime}\right)}= \\
& =-\frac{i s_{y} \sin \pi \nu e^{i\left(\theta^{\prime}-\theta\right) / 2}}{\sqrt{\sinh \gamma_{\theta} \sinh \gamma_{\theta^{\prime}}}} \frac{\vartheta_{2}(0 \mid \tau) \vartheta_{4}(0 \mid \tau)}{\vartheta_{3}(0 \mid \tau) \vartheta_{3}(\pi \nu \mid \tau)} \frac{\vartheta_{2}\left(x_{\theta}+x_{\theta^{\prime}}+\pi \nu \mid \tau\right)}{\vartheta_{4}\left(x_{\theta}+x_{\theta^{\prime}} \mid \tau\right)}
\end{aligned}
$$

The limit of multiparticle form factors can be found in a similar way: it suffices to remove the functions $\eta_{\theta}$ from (46) and to replace $X_{\alpha^{\prime}}-X_{\alpha}$ in (49) by $\pi \nu$.

In the case $\nu= \pm \frac{1}{2}$, the formulas (54)-(56) reduce to simpler expressions

$$
\begin{aligned}
& \left\langle\mathcal{O}_{ \pm \frac{1}{2}}\right\rangle=\left(1-k^{2}\right)^{\frac{1}{4}}, \\
& \mathbf{F}_{ \pm \frac{1}{2}}\left(\theta ; \mid \theta^{\prime} ;\right)=\frac{i e^{i\left(\theta^{\prime}-\theta\right) / 2}}{\sqrt{\sinh \gamma_{\theta} \sinh \gamma_{\theta^{\prime}}}} \frac{\sinh \frac{\gamma_{\theta}+\gamma_{\theta^{\prime}}}{2}}{\sin \frac{\theta-\theta^{\prime}}{2}}, \\
& \mathbf{F}_{ \pm \frac{1}{2}}\left(\theta ; \theta^{\prime} \mid ;\right)=\frac{-i s_{x}^{*} s_{y} e^{i\left(\theta^{\prime}-\theta\right) / 2}}{\sqrt{\sinh \gamma_{\theta} \sinh \gamma_{\theta^{\prime}}}} \frac{\sin \frac{\theta+\theta^{\prime}}{2}}{\sinh \frac{\gamma_{\theta}+\gamma_{\theta^{\prime}}}{2}} .
\end{aligned}
$$

They can be proved using the relations $\vartheta_{1}\left(z+\frac{\pi}{2}\right)=\vartheta_{2}(z), \vartheta_{3}\left(z+\frac{\pi}{2}\right)=\vartheta_{4}(z)$, rewriting the theta function ratios in terms of elliptic functions and finally passing to the trigonometric parametrization.

\subsection{Scaling limit}

The gap in the energy spectrum closes as $\gamma_{0} \rightarrow 0$. Since $\gamma_{0}=2\left(\mathcal{K}_{y}-\mathcal{K}_{x}^{*}\right)$, this corresponds to $k \rightarrow 1$ (or, equivalently, $\tau \rightarrow i 0$ or $q \rightarrow 1$ ) in terms of elliptic parameters. Also note that

$$
-\tau^{-1}=\frac{i K}{K^{\prime}}=-\frac{i}{\pi} \ln \frac{1-k^{2}}{16}+o(1) \quad \text { as } k \rightarrow 1 .
$$

In order to obtain the asymptotics of the vacuum expectation value (54) in the vicinity of the critical point, recall the modular transformation

$$
\vartheta_{3}(z \mid \tau)=(-i \tau)^{-1 / 2} e^{-\frac{i z^{2}}{\pi \tau}} \vartheta_{3}\left(-\frac{z}{\tau} \mid-\frac{1}{\tau}\right)
$$

and the product formula

$$
\vartheta_{3}(z \mid \tau)=\prod_{j=1}^{\infty}\left(1-q^{2 j}\right)\left(1+q^{2 j-1} e^{2 i z}\right)\left(1+q^{2 j-1} e^{-2 i z}\right) .
$$

Rewrite the theta functions in (54) using (61). The nome of the transformed functions vanishes as $k \rightarrow 1$. The representation (62) therefore implies that

$$
\lim _{\tau \rightarrow i 0} \vartheta_{3}\left(-\frac{\pi \nu}{\tau} \mid-\frac{1}{\tau}\right)= \begin{cases}1 & \text { for }|\nu|<\frac{1}{2} \\ 2 & \text { for } \nu= \pm \frac{1}{2}\end{cases}
$$


This in turn can be used together with (60) to show that, as $k \rightarrow 1$,

$$
\left\langle\mathcal{O}_{\nu}\right\rangle=\left\{\begin{aligned}
2^{-4 \nu^{2}}\left(1-k^{2}\right)^{\nu^{2}}[1+o(1)] & \text { for }|\nu|<\frac{1}{2}, \\
\left(1-k^{2}\right)^{\frac{1}{4}}[1+o(1)] & \text { for } \nu= \pm \frac{1}{2} .
\end{aligned}\right.
$$

The second approximation is in fact exact, cf. (57). As the r.h.s. of (54) is periodic in $\nu$ with period 1, the critical asymptotics of the vacuum expectation value for any $\nu$ can be deduced from (63). Note that the scaling dimension of the twist field for $|\nu| \leq \frac{1}{2}$ has the expected value $\nu^{2}$.

In the vicinity of the critical point, our initial lattice model becomes equivalent to a field theory of free massive Dirac fermions. Correlation functions of twist fields in this theory are determined by momenta at the scale of inverse correlation length (for convenience, we change the domain of definition of lattice momenta from $[0,2 \pi]$ to $[-\pi, \pi])$.

More formally, denote $\varepsilon=\frac{1-k^{2}}{2 c_{y}}$, set $\theta=\varepsilon \sinh \xi$ and let $k \rightarrow 1$. The dispersion relation (3) then implies that $\gamma_{\theta} \rightarrow \varepsilon s_{y} \cosh \xi$. One also has

$$
-\frac{x_{\theta}-x_{\theta^{\prime}}}{\tau} \rightarrow \frac{i\left(\xi-\xi^{\prime}\right)}{2} \text {. }
$$

Normalized scaled two-particle form factors of twist fields are determined by

$$
\begin{aligned}
& \mathbb{F}_{\nu}\left(\xi ; \mid \xi^{\prime} ;\right)=\lim _{k \rightarrow 1} \varepsilon \mathbf{F}_{\nu}\left(\varepsilon \sinh \xi ; \mid \varepsilon \sinh \xi^{\prime} ;\right), \\
& \mathbb{F}_{\nu}\left(\xi ; \xi^{\prime} \mid ;\right)=\lim _{k \rightarrow 1} \varepsilon \mathbf{F}_{\nu}\left(\varepsilon \sinh \xi ;-\varepsilon \sinh \xi^{\prime} \mid ;\right) .
\end{aligned}
$$

where the variables $\xi, \xi^{\prime}$ have the meaning of particle rapidities.

To compute the corresponding limits, one can adopt the same approach as in the above asymptotic analysis of the VEV. Transform the theta functions in (55) -(56) using Jacobi's imaginary transformations, rewrite the result using product formulas, and then let $\tau \rightarrow i 0$. For $|\nu|<\frac{1}{2}$, one finds

$$
\begin{aligned}
& \mathbb{F}_{\nu}\left(\xi ; \mid \xi^{\prime} ;\right)=\frac{\sin \pi \nu}{\sqrt{\cosh \xi \cosh \xi^{\prime}}} \frac{e^{\nu\left(\xi^{\prime}-\xi-i \pi\right)}}{\sinh \frac{\xi^{\prime}-\xi}{2}}, \\
& \mathbb{F}_{\nu}\left(\xi ; \xi^{\prime} \mid ;\right)=\frac{\sin \pi \nu}{\sqrt{\cosh \xi \cosh \xi^{\prime}}} \frac{i e^{\nu\left(\xi^{\prime}-\xi\right)}}{\cosh \frac{\xi^{\prime}-\xi}{2}} .
\end{aligned}
$$

This reproduces two-particle (and hence all) form factors of the continuum twist fields in the massive Dirac theory [1, 23, 29, 31, which correspond to the exponential fields in the sine-Gordon model at the free-fermion point.

Likewise, it can be deduced from (58)-(59) that for $\nu= \pm \frac{1}{2}$ one has

$$
\begin{aligned}
& \mathbb{F}_{ \pm \frac{1}{2}}\left(\xi ; \mid \xi^{\prime} ;\right)=\frac{1}{2}\left[\mathbb{F}_{\frac{1}{2}-0}\left(\xi ; \mid \xi^{\prime} ;\right)+\mathbb{F}_{-\frac{1}{2}+0}\left(\xi ; \mid \xi^{\prime} ;\right)\right]=\frac{-i \operatorname{coth} \frac{\xi^{\prime}-\xi}{2}}{\sqrt{\cosh \xi \cosh \xi^{\prime}}}, \\
& \mathbb{F}_{ \pm \frac{1}{2}}\left(\xi ; \xi^{\prime} \mid ;\right)=\frac{1}{2}\left[\mathbb{F}_{\frac{1}{2}-0}\left(\xi ; \xi^{\prime} \mid ;\right)+\mathbb{F}_{-\frac{1}{2}+0}\left(\xi ; \xi^{\prime} \mid ;\right)\right]=\frac{i \tanh \frac{\xi^{\prime}-\xi}{2}}{\sqrt{\cosh \xi \cosh \xi^{\prime}}} .
\end{aligned}
$$


The fact that $\mathbb{F}_{\frac{1}{2}-0} \neq \mathbb{F}_{-\frac{1}{2}+0}$ can be understood as follows. Monodromy conditions for fermion fields in the continuum give a system of integral equations for the two-particle form factors [1]. This system has a unique admissible solution for $|\nu|<\frac{1}{2}$, and two solutions for $\nu= \pm \frac{1}{2}$. Each of the two solutions gives rise to a twist field operator. Any linear combination of these operators leads to the required fermion branching.

\section{Concluding remarks}

Finite-lattice form factors in the conventional free-fermion models (triangular Ising lattice, XY quantum spin chain, $\mathrm{BBS}_{2}$ model) can be obtained [12, 14] from those of the Ising spin on the square lattice [3, 4, 9, 10, 13. The fields considered in this paper are more general; in particular, their scaling dimension continuously depends on a real parameter $\nu$. We compute their form factors explicitly in terms of the Jacobi theta functions and show that in the scaling limit they reduce to form factors of the exponential fields of the sine-Gordon model at the free-fermion point.

Determinantal form of form factors is also encountered in some of the interacting integrable models, see e. g. Slavnov's formula for scalar products of Bethe states in the spin- $\frac{1}{2}$ XXZ chain [17, 32]. An intriguing question is therefore if it is possible to go beyond the free-fermion point and calculate form factors of twist fields in the integrable lattice regularization of the massive Thirring model [22] (related to the eight-vertex statistical model). We believe that the present work makes a step in this direction.

Another challenge is to complete form factor derivation for $\mathbb{Z}_{N \text {-symmetric }}$ superintegrable chiral Potts quantum chain. It was recently shown that they have Ising form up to unknown scalar factors labeled by the pairs of the so-called Onsager sectors [15. Putative free-fermion part of the integrable structure of this model is yet to be elucidated.

\section{Acknowledgments}

The authors are grateful to S. Pakuliak and V. Shadura for useful discussions. This work was partially supported by the Program of Fundamental Research of the Physics and Astronomy Division of NASU, by the joint Ukrainian-Russian SFFR-RFBR project F40.2/108, the joint PICS project of CNRS and NASU, and IRSES project "Random and Integrable Models in Mathematical Physics".

\section{References}

[1] Bernard D and LeClair A 1994 Differential equations for sine-Gordon correlation functions at the free fermion point Nucl. Phys. B426 534-558 arXiv:hep-th/9402144v2

[2] Borodin A and Deift P 2002 Fredholm determinants, Jimbo-Miwa-Ueno tau-functions, and representation theory Comm. Pure Appl. Math. 55 1160-1230 arXiv:math-ph/0111007v1

[3] Bugrij A I and Lisovyy O 2003 Spin matrix elements in 2D Ising model on the finite lattice Phys. Letts. A319 390-394 arXiv:0708.3625 [nlin.SI] 
Form factors of twist fields in the lattice Dirac theory

[4] Bugrij A I and Lisovyy O 2004 Correlation function of the two-dimensional Ising model on a finite lattice. II Theor. Math. Phys. 140 987-1000 arXiv:0708.3643 [nlin.SI]

[5] Bugrij A I and Shadura V N 1999 Asymptotic expression for the correlation function of twisted fields in the two-dimensional Dirac model on a lattice Theor. Math. Phys. 121 1535-1549 arXiv:hep-th/9907040v3

[6] Doyon B 2003 Two-point correlation functions of scaling fields in the Dirac theory on the Poincaré disk Nucl.Phys. B675 607-630 arXiv:hep-th/0304190v2

[7] Doyon B and Fonseca P 2004 Ising field theory on a pseudosphere J. Stat. Mech. 0407 P07002 arXiv:hep-th/0404136v1

[8] Doyon B and Silk J 2011 Correlation functions of twist fields from Ward identities in the massive Dirac theory J. Phys. A44 295402 arXiv:1103.2328v1 [hep-th]

[9] von Gehlen G, Iorgov N, Pakuliak S, Shadura V and Tykhyy Yu 2007 Form-factors in the Baxter-Bazhanov-Stroganov model I: Norms and matrix elements J. Phys. A40 14117-14138 arXiv:0708.4342 [nlin.SI]

[10] von Gehlen G, Iorgov N, Pakuliak S, Shadura V and Tykhyy Yu 2008 Form-factors in the Baxter-Bazhanov-Stroganov model II: Ising model on the finite lattice J. Phys. A41 095003 arXiv:0711.0457 [nlin.SI]

[11] Hystad G 2011 Periodic Ising correlations J. Math. Phys. 52013302 arXiv:1011.2223v1 [math-ph]

[12] Iorgov N 2011 Form-factors of the finite quantum XY-chain J. Phys. A44 335005 arXiv:0912.4466 2 [cond-mat.stat-mech]

[13] Iorgov N and Lisovyy O 2011 Ising correlations and elliptic determinants J. Stat. Phys. 143 33-59 arXiv:1012.2856v2 [math-ph]

[14] Iorgov N and Lisovyy O 2011 Finite-lattice form factors in free-fermion models J. Stat. Mech. P04011 arXiv:1102.2145v2 [cond-mat.stat-mech]

[15] Iorgov N, Pakuliak S, Shadura V, Tykhyy Yu and von Gehlen G 2010 Spin operator matrix elements in the superintegrable chiral Potts quantum chain J. Stat. Phys. 139 743-768 arXiv:0912.5027 [cond-mat.stat-mech]

[16] Kadanoff L P and Ceva H 1971 Determination of an operator algebra for the two-dimensional Ising model Phys. Rev. B3 3918-3939

[17] Kitanine N, Maillet J M and Terras V 1998 Form factors of the XXZ Heisenberg spin-1/2 finite chain Nucl. Phys. B554 647-678 arXiv:math-ph/9807020

[18] Lisovyy O 2008 On Painlevé VI transcendents related to the Dirac operator on the hyperbolic disk J. Math. Phys. 49 093507 arXiv:0710.5744v1 [math-ph]

[19] Lisovyy O 2011 Dyson's constant for the hypergeometric kernel New trends in quantum integrable systems : proceedings of the Infinite Analysis 09 ed B Feigin, M Jimbo and M Okado (Singapore: World Scientific) pp 243-267 arXiv:0910.1914 2 [math-ph]

[20] Lisovyy O 2009 Finite-volume correlation functions of monodromy fields on the lattice: Toeplitz representation Systèmes intégrables et Théorie des champs quantiques ed P Baird, F Helein, J Kouneiher, F Pedit and V Roubtsov (Paris: Hermann) pp 170-186.

[21] Lukyanov S and Zamolodchikov A 1997 Exact expectation values of local fields in the quantum sine-Gordon model Nucl. Phys. B493 571-587 arXiv:hep-th/9611238

[22] Lüscher M 1976 Dynamical charges in the quantized renormalized massive Thirring model Nucl. Phys. B117 475-492

[23] Marino E C, Schroer B and Swieca J A 1982 Euclidean functional integral approach for disorder variables and kinks Nucl. Phys. B200 473-497

[24] Palmer J 2007 Planar Ising correlations (Prog. Math. Phys. 49 Boston: Birkhäuser)

[25] Palmer J 1985 Monodromy fields on $\mathbb{Z}_{2}$ Comm. Math. Phys. 102 175-206

[26] Palmer J 1986 Critical scaling for monodromy fields Comm. Math. Phys. 104 353-385

[27] Palmer J, Beatty M and Tracy C A 1994 Tau functions of the Dirac operator on the Poincaré disk Comm. Math. Phys. 165 97-173 arXiv:hep-th/9309017v1

[28] Palmer J and Hystad G 2010 Spin matrix for the scaled periodic Ising model J. Math. Phys. 51 
123301 arXiv:1008.0352 2 [nlin.SI]

[29] Sato M, Miwa T and Jimbo M 1979 Holonomic quantum fields III-IV Publ. RIMS Kyoto Univ. 15 577-629; 15 871-972

[30] Schroer B and Truong T T 1978 The relativistic quantum fields of the D=2 Ising model Phys. Letts. B72 371-374

[31] Schroer B and Truong T T 1978 The order/disorder quantum field operators associated with the two-dimensional Ising model in the continuum limit Nucl. Phys. B144 80-122

[32] Slavnov N 1989 Calculation of scalar products of wave functions and form factors in the framework of the algebraic Bethe ansatz Theor. Math. Phys. 79 502-508 